\title{
RESEARCH
}

Open Access

\section{Development and validation of the Axillary Sweating Daily Diary: a patient-reported outcome measure to assess axillary sweating severity}

\author{
L. M. Nelson ${ }^{1 *}$, D. DiBenedetti ${ }^{1}$, D. M. Pariser², D. A. Glaser ${ }^{3}$, A. A. Hebert ${ }^{4}$, H. Hofland ${ }^{5}$, J. Drew ${ }^{5}$, D. Ingolia ${ }^{5}$, \\ K. K. Gillard ${ }^{5}$ and S. Fehnel ${ }^{1}$
}

\begin{abstract}
Background: Hyperhidrosis is estimated to affect $\sim 4.8 \%$ of the US population, and most patients experience a negative psychological impact. Here, we describe development and psychometric evaluation of a patient-reported outcome (PRO) measure to assess severity of axillary hyperhidrosis in clinical trials that meets current U.S. regulatory standards to support product approvals.

Methods: Three rounds of hybrid concept-elicitation/cognitive-debriefing qualitative interviews were conducted in adults with clinician-diagnosed primary axillary hyperhidrosis, followed by similar interviews in children/adolescents. The draft measure included diary items for presence, severity, impact and bothersomeness (basis of the Axillary Sweating Daily Diary [ASDD]), exploratory weekly impact items, and a single-item Patient Global Impression of Change (PGIC). Phase 2 (adults only) and phase 3 (adults and children $\geq 9$ years) clinical trial data were utilized to evaluate measurement properties of the resulting draft measure: floor/ceiling effects, nonresponse bias, test-retest reliability, construct validity, and responsiveness were assessed. The primary concept of interest was axillary sweating severity (ASDD Item 2); however, additional supportive concepts were explored to allow for development of a comprehensive hyperhidrosis measure.

Results: Twenty-nine patient interviews were conducted ( $N=21$ adult and $N=8$ children/adolescents), resulting in the ASDD ( 4 items, patients $\geq 16 y$ ) and child-specific ASDD-C ( 2 items $\geq 9 y$ to $<16 y$ ), as well as 6 Weekly Impact items and the PGIC (patients $\geq 16 y$ ). No floor/ceiling effects or response biases were identified. Consistency between hypothesized and observed correlation patterns between ASDD/ASDD-C items and other efficacy measures supported construct validity. Intraclass correlation coefficients supported test-retest reliability (0.91-0.93; Item 2). Large effect sizes ( -2.2 to -2.4$)$ demonstrated that the ASDD/ASDD-C Item 2 could detect changes in hyperhidrosis severity, supporting the measure's responsiveness. Patients perceiving a moderate improvement in symptoms on the PGIC experienced an average 3.8-point improvement on ASDD axillary sweating severity (Item 2); thus, a 4-point responder threshold was defined as a clinically meaningful change.

Conclusions: Qualitative and quantitative evidence support the reliability and validity of the ASDD/ASDD-C and its use in the clinical evaluation of axillary hyperhidrosis treatments. Further evaluation of this measure in future research studies is warranted to demonstrate consistent performance across different axillary hyperhidrosis populations and in different study contexts.
\end{abstract}

Keywords: Hyperhidrosis, Sweating severity, Patient reported outcome, Axillary sweating daily diary (ASDD)

\footnotetext{
* Correspondence: Inelson@rti.org

${ }^{1}$ RTI Health Solutions, 200 Park Offices Drive, Research Triangle Park, NC 27709, USA

Full list of author information is available at the end of the article
} 


\section{Background}

Hyperhidrosis $(\mathrm{HH})$ is a condition in which sweat production exceeds that which is physiologically necessary to maintain thermal homeostasis [1]. This burdensome condition is estimated to affect up to $4.8 \%$ of the U.S. population, or 15.3 million Americans, and causes substantial impairment in patient daily life, as approximately three-quarters of patients report negative impacts on their social life, sense of well-being, and/or mental health [2].

A number of tools have been utilized to measure patient-reported severity, impact, and bother associated with hyperhidrosis [3]. Generic health-related quality of life (HRQOL) measures such as the Short Form Health Surveys (SF-36 and SF-12) and the Nottingham Health Profile (NHP) have been used [4, 5]; however, these cover broader constructs of health, do not necessarily reflect areas of functioning that are particularly relevant to patients with hyperhidrosis, and lack sensitivity needed to discern clinically meaningful treatment benefit [6] Similarly, dermatology-specific measures (Skindex, Dermatology Life Quality Index [DLQI]) have been administered to patients with hyperhidrosis but were not developed to capture all relevant hyperhidrosis-specific concepts [7-9]. The most commonly used hyperhidrosis-specific scale is the Hyperhidrosis Disease Severity Scale (HDSS). Although this scale has been broadly adopted in clinical practice to evaluate disease severity, it combines two distinct concepts in the same item (ie, tolerability and interference with daily activities), thus limiting interpretation of study results. In addition, patient input was not solicited during the development of the HDSS [10, 11]. Other diseasespecific measures include the Hyperhidrosis Impact Questionnaire (HHIQ) [12], the Amir-de Campos Clinical Protocol for QoL [13, 14], and the Keller scale [15]. The Hyperhidrosis Quality of Life Index (HidroQOL@) is a more recently developed measure; however, at the time this research was designed and conducted, it was not publicly available and its measurement properties had not been evaluated in an interventional trial setting $[16,17]$.

Although the instruments described above have been used to better characterize patients with hyperhidrosis, it is unclear whether any were developed to support product approvals in accordance with most recent U.S. regulatory standards for PRO measures (ie, for inclusion as key endpoint(s) in a clinical trial) [18]. This prompted the development of a new PRO to measure hyperhidrosis treatment benefit to meet U.S. registration needs, and to more broadly understand the impact of hyperhidrosis on patients' lives.

Here, we describe the development, features, and validation of the Axillary Sweating Daily Diary (ASDD) and Axillary Sweating Daily Diary-Children (ASDD-C), with a focus on the psychometric evaluation of axillary sweating severity item (Item 2).

\section{Methods \\ Instrument development}

A brief overview of the instrument development process is detailed in Fig. 1. Specifically, one-on-one hybrid conceptelicitation/cognitive-debriefing qualitative interviews were conducted with patients $\geq 18$ years with cliniciandiagnosed primary axillary hyperhidrosis $(N=21)$ in 3 different geographic locations between January 2014 and February 2014. Interview results were qualitatively analyzed in three sets, where each set of interviews built upon the findings of previous interviews to confirm the adequacy of modifications and allow testing of any new items. Consistent with the inclusion criteria for the initial dose-finding trials, all participants were required to exhibit hyperhidrosis symptomology for at least 6 months, as well as report an HDSS score of 3 or 4, indicating "barely tolerable" or "intolerable" sweating and sweating "frequently" or "always" interfering with daily activities. Preliminary concepts were selected for inclusion in a draft item pool based on clinical expertise, review of the literature, and review of other questionnaires (eg, HDSS, questions on the International Hyperhidrosis Society website [www. sweathelp.org]). Given that the primary concept of measurement, sweating severity, was both straightforward and clearly important within the context of hyperhidrosis, draft items addressing this concept were developed prior to patient engagement and were comprised of daily diary items covering concepts pertaining to presence, severity, impact and bother of axillary hyperhidrosis (ultimately referred to as the ASDD). Additional supportive concepts were selected for inclusion in a preliminary item pool to further explore the impact of axillary hyperhidrosis on various areas of patients' lives (ultimately referred to as the Weekly Impact Items). A Patient Global Impression of Change (PGIC) item was also included to assess patientperceived changes in underarm sweating and facilitate the interpretation of change scores on the diary items. Multiple questions were drafted for each concept so variable wording and response scales could be tested during patient interviews. All interviews were conducted by two experienced qualitative researchers according to a semistructured interview guide created collaboratively by the developers. Each interview began with open-ended concept elicitation. Participants were asked to describe their experiences with axillary sweating, including variations in severity, the extent and situations in which they were bothered by their sweating, and how their lives were impacted by hyperhidrosis. Following the concept elicitation phase, the same patients were then asked to provide feedback on the draft items via paper forms during a cognitive debriefing phase to support the salience and importance 


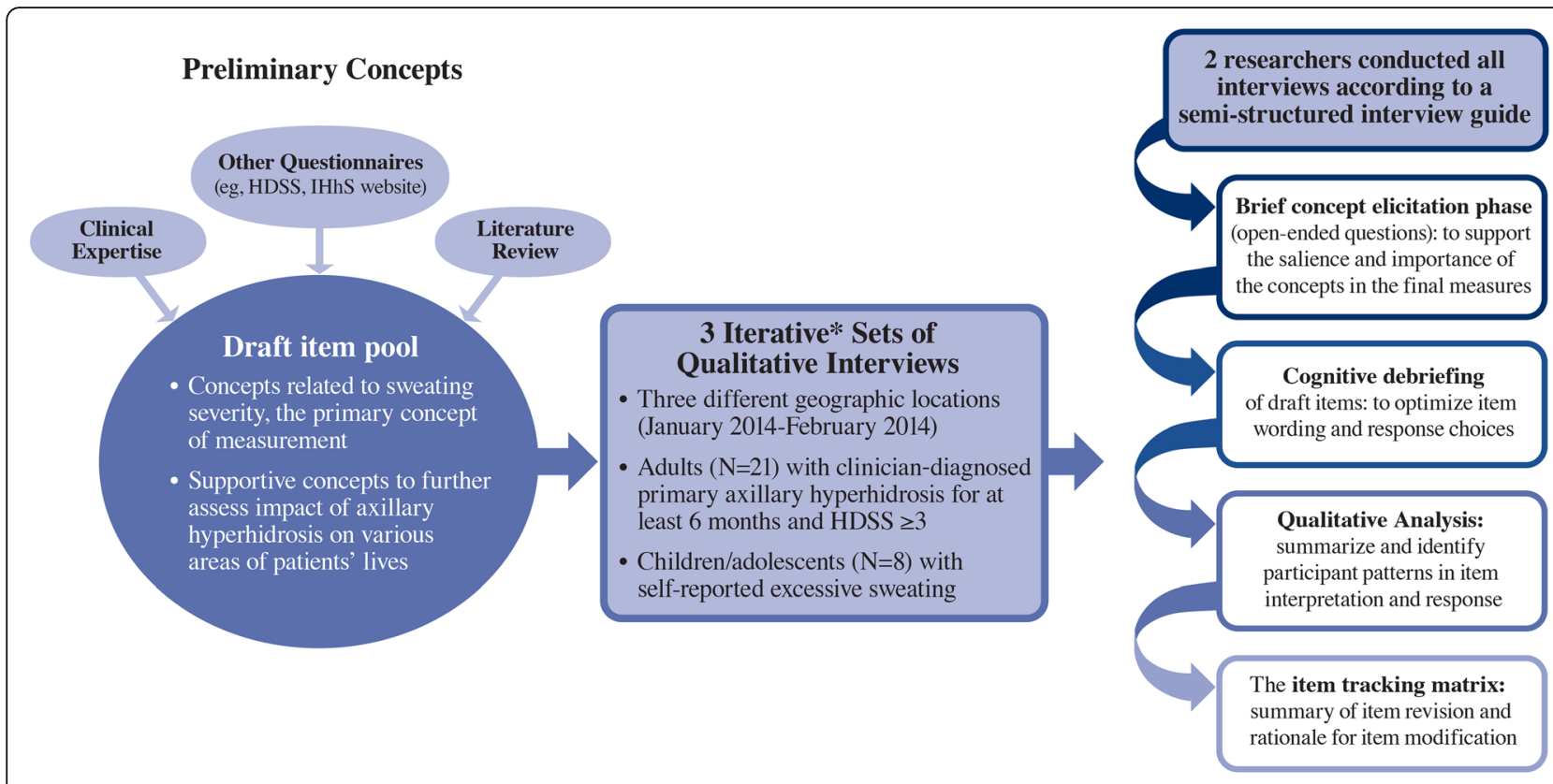

*each set of interviews built upon the findings of previous interviews to confirm adequacy of modifications and allow testing of any new items

Fig. 1 Overview of the Instrument Development Process. ASDD, Axillary Sweating Daily Diary; HDSS, Hyperhidrosis Disease Severity Scale; IHhS, International Hyperhidrosis Society

of the concepts, as well as to optimize item wording and response options. Specifically, participants were asked to respond to each item while thinking aloud. Interviews were reviewed individually and compared to other interviews in order to summarize and identify participant patterns in item interpretation and response. An item tracking matrix was created, which included any revisions made to an item and a rationale for the item modification. Additional follow-up questions were also posed by the interviewers to further elucidate comprehension and response processes. If concept saturation was not reached following qualitative data analysis based on the findings during each set of interviews, additional interviews were to be conducted with new patients.

Following the decision to include individuals as young as 9 years of age in a future clinical trial program, a similar set of qualitative interviews was conducted with children and adolescents < 18 years with self-reported excessive sweating $(N=8)$ to evaluate whether the new measure would be relevant for children $\geq 9$ years of age. While the sample size was deemed adequate to achieve concept saturation, if new concepts had been generated during these interviews, testing with additional pediatric patients would have been conducted.

\section{Psychometric evaluation}

\section{Phase 2 and 3 study design and analysis}

To further support use of the ASDD/ASDD-C axillary sweating severity item (Item 2) in clinical trials evaluating the efficacy of new treatments for hyperhidrosis, the measurement properties of ASDD Item 2 were evaluated using data from a 4-week phase 2 trial of glycopyrronium tosylate $(\mathrm{GT})$ in patients $\geq 18$ years of age (study DRM04-HH02, NCT02129660; $N=102$ ) [19]. Phase 2 data were also used to establish basic psychometric properties of ASDD impact and bothersomeness items (Items 3 and 4). Weekly Impact items, PGIC, HDSS, and gravimetric data were also collected as study outcomes.

Additional psychometric evaluation of ASDD/ASDD-C Item 2 was subsequently conducted based on pooled data from two, 4-week phase 3 trials of GT, ATMOS-1 (DRM04-HH04, NCT02530281; $N=344 ;$ sites in the U.S. and Germany) and ATMOS-2 (DRM04-HH05, NCT02530294; $N=353$; U.S. sites only) [20]. Study designs and inclusion criteria were similar for the phase 2 and phase 3 studies; the phase 3 study also included the Dermatology Life Quality Index (DLQI) and Children's Dermatology Life Quality Index (C-DLQI, for ages 4 to 16 years) in addition to the other outcomes collected and described above for phase 2. The DLQI/C-DLQI is a dermatology-specific, health-related quality of life questionnaire developed to understand the impacts of skin conditions on patients' lives $[9,21]$. Eligible patients were $\geq 18$ years of age for phase 2 and $\geq 9$ years of age for the phase 3 studies; had a diagnosis of primary axillary hyperhidrosis for $\geq 6$ months; had HDSS grade 3 or 4 ; had sweat production $\geq 50 \mathrm{mg} /$ axilla/ $5 \mathrm{~min}$ while at rest 
Table 1 Axillary Hyperhidrosis Patient Measures (AHPM)

Axillary Sweating Daily Diary (ASDD)
Instructions: The questions in the diary are designed to measure the
severity and impact of any underarm sweating you have experienced
within the previous 24-h period, including nighttime hours. While you
may also experience sweating in other locations on your body, please
be sure to think only about your underarm sweating when answering
these questions.
$\begin{array}{r}\text { Please complete the diary each evening before you go to sleep. } \\ \text { Item } 1 \text { [Gatekeeper] } \\ \text { During the past } 24 \text { h, did you } \\ \text { have any underarm sweating? } \\ \text { Yes/No } \\ \text { When Item } 1 \text { is answered "no," } \\ \text { Item } 2 \text { is skipped and scored as } \\ \text { zero }\end{array}$

Item 2

During the past $24 \mathrm{~h}$, how would you rate your underarm sweating at its worst?

0 (no sweating at all) to 10 (worst possible sweating)

Item 3

During the past $24 \mathrm{~h}$, to what extent did your underarm sweating impact your activities?

0 (not at all), 1 (a little bit), 2 (a

moderate amount), 3 (a great deal),

4 (an extreme amount)

Item 4

During the past $24 \mathrm{~h}$, how bothered were you by your underarm sweating? 0 (not at all bothered), 1 (a little bothered), 2 (moderately bothered), 3 (very bothered), 4 (extremely bothered)

Axillary Sweating Daily Diary-Children (ASDD-C) ${ }^{b}$

Instructions: These questions measure how bad your underarm sweating was last night and today. Please think only about your underarm sweating when answering these questions.

Please complete these questions each night before you go to sleep.

Item 1 [Gatekeeper]

Thinking about last night and today, did you have any underarm sweating?

Yes/No

When Item 1 is answered "no," Item 2 is skipped and scored as zero

Item 2 Thinking about last night and today, how bad was your underarm sweating? 0 (no sweating at all) to 10 (worst possible sweating)

Weekly Impact Items ${ }^{\mathrm{a}}$

Instructions: Please respond "Yes" or "No" to each of the following questions.

\footnotetext{
a. During the past 7 days, did you Yes/No

ever have to change your shirt

during the day because of your

underarm sweating?
}

b. During the past 7 days, did

Yes/No

you ever have to take more than

1 shower or bath a day because

of your underarm sweating?
Table 1 Axillary Hyperhidrosis Patient Measures (AHPM) (Continued)

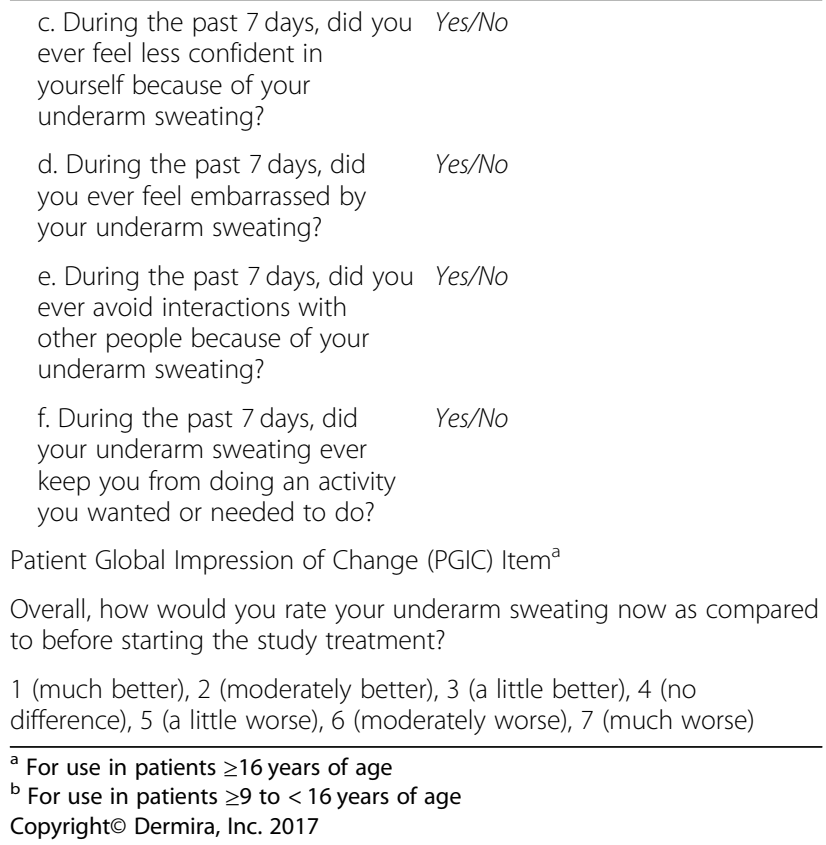

at room temperature; and for phase 3 studies, had weekly average ASDD Item 2 scores $\geq 4$.

PRO measures were completed by patients using an electronic tablet device provided to them during the study period as follows: for patients $\geq 16$ years of age, the ASDD was completed daily (before bed) for 4 to 7 days during screening ( 7 to 10 days prior to randomization) and during treatment; in the phase 3 studies, patients < 16 years completed the ASDD-C items (Items 1 and 2 only) according to the same schedule (phase 3 only). Patients $\geq 16$ years of age additionally completed two other measures developed alongside the ASDD, namely the Weekly Impact items (Table 1) and the single-item PGIC (Table 1). These 3 measures (the ASDD, the Weekly Impact items, and the PGIC) are collectively referred to in Table 1 as the Axillary Hyperhidrosis Patient Measures (AHPM). The HDSS and Weekly Impact items were completed at Baseline and each week during the treatment period; the DLQI (phase 3 only) and PGIC was completed at Week 4 or at the end of treatment (ie, early termination). The ASDD/ASDD-C, Weekly Impact items, and PGIC were completed via electronic data capture; the HDSS and DLQI/C-DLQI (phase 3 only) were completed weekly on paper. Sweat production, assessed gravimetrically, was measured for the right and left axilla at Baseline and each weekly clinic visit.

ASDD/ASDD-C items were scored as a weekly average of daily responses; at least 4 days of daily data were required for analysis. Responses to the 6 Weekly Impact items were summed such that a response of "Yes" to any of the items resulted in a score of 1 per item. This was 
referred to as the Weekly Impact summary (possible score range of 0 to 6) and used in exploratory analyses. All statistical tests were two-tailed using a type I error rate of $5 \%$ (alpha $=0.05)$. Rather than relying on statistical significance alone, the interpretation of effect size estimates and patterns of results were emphasized. Observed values were used for all calculations for the phase 2 study. For ASDD Item 2 in the phase 3 studies, the weekly mean from Markov Chain Monte Carlo (MCMC) imputation was used; observed values were used for ASDD Items $3 \& 4$ as well as the PGIC. For binary response data (i.e., ASDD/ASDD-C Item 1, Weekly Impact items), missing values were considered a "No" response.

\section{Evaluation of measurement properties}

Potential floor and ceiling effects (approximately double the expected proportion given equal distribution across response options or $\geq 20 \%$ of sample responding at either extreme of the scale), were evaluated based on responses during screening (Baseline) using both summary statistics and graphical techniques. Test-retest reliability for Item 2, 3, and 4 was evaluated to assess each item's stability through the computation of intraclass correlation coefficients (ICCs) between Week 3 and Week 4, using a threshold of $\geq 0.70$ [22]. Week 3 and Week 4 were chosen as they represented time points at which symptoms were considered to be most stable following initiation of treatment.

Construct validity was evaluated at Week 4 based on correlations between ASDD/ASDD-C Item 2 gravimetric measurements and established PRO measures (HDSS and DLQI). Week 4 correlations were also explored between ASDD/ASDD-C Item 2 and ASDD Items 3 and 4, the Weekly Impact items, and the PGIC [23]. Correlations $\geq 0.50$ were considered strong, $\geq 0.30$ to $<0.50$ moderate, and $<0.30$ weak [24].Correlations with items addressing related constructs (Items 3 and 4) were hypothesized to yield larger correlations as compared with those computed between items addressing more disparate constructs: given that sweating can be episodic and can vary based on time of day, emotional stimuli, and/or daily activities [25], weak positive correlations were expected between ASDD/ASDD-C Item 2 scores and gravimetric measurements. Weak to moderate correlations were expected between DLQI and ASDD/ ASDD-C Item 2, as DLQI may capture some, but not all, hyperhidrosis-specific issues. The HDSS was expected to correlate moderately to strongly with ASDD/ ASDD-C Item 2 as both items are disease-specific, although the correlations could be mitigated due to the limitations in HDSS described above.

Known-groups validity (the ability of the measure to discriminate between clinically distinct subgroups) was evaluated at Week 4 based on two-sample t-tests comparing differences in scores on ASDD/ASDD-C Item 2 between subgroups predefined based on HDSS grades (score of 1 vs score of 3 or 4), DLQI score ranges (range of $0-10$ and $0-12$ for no to moderate impact for DLQI and CDLQI, respectively, and range of 21-30 and 19-20 for large to extremely large impact for DLQI and CDLQI, respectively) $[9,21,26,27]$, and gravimetricallymeasured sweat production (1st vs 4th distribution quartiles).

Responsiveness was evaluated through effect size computation between Baseline and Week 4 to evaluate the ability of the ASDD/ASDD-C items to detect change. A responder definition to establish a threshold for a magnitude of within-person change considered to be clinically meaningful was determined based on methods outlined in the FDA PRO guidance [18], which recommends the use of self-reported retrospective measures of change as external anchors for the estimation of meaningful change. Such an anchor must be both a valid measure of change and easier to interpret than the PRO measure [28]. Thus, the PGIC was utilized as the predefined anchor. Specifically, responders were defined as patients who rated their underarm sweating as "moderately better" on the PGIC compared with the start of study treatment. Exploratory within-person changes in HDSS scores were also calculated against PGIC responses. Finally, although PGIC measures are widely accepted as an appropriate anchor [28], exploratory within-person change scores on the ASDD were calculated across different magnitudes of change in HDSS scores.

\section{Results}

\section{Instrument development}

The cohort of adult interviewees was predominantly white $(76 \%)$ and female (76\%) (Table 2). In general, adult interviewees reported that the most relevant diary items intended for daily administration as part of the ASDD included those related to the presence, severity, overall impact, and bother associated with hyperhidrosis. Participants noted that the 24-h reference period was appropriate. Several of the items originally proposed for inclusion in the ASDD were considered to be salient to some patients at some points in time (interference with activities, need for additional showers/baths, and need for additional shirt changes); thus, they were better suited for inclusion in the Weekly Impact items (7-day recall) and tested as such in subsequent rounds of interviews. Additional concepts tested and retained as a component of the Weekly Impact items included avoiding social interactions, confidence and embarrassment. Of note, all adult interviewees reported embarrassment due to their axillary hyperhidrosis and nearly all (95\%) modified clothing choices due to their symptoms (Fig. 2). 
Table 2 Characteristics of Interviewees Reported at Screening

\begin{tabular}{|c|c|c|}
\hline Characteristic & $\begin{array}{l}\text { Adults }(N= \\
21)\end{array}$ & $\begin{array}{l}\text { Pediatric } \\
(N=8)\end{array}$ \\
\hline \multicolumn{3}{|c|}{ Excessive sweating locations, $\mathrm{n}(\%)^{\mathrm{a}}$} \\
\hline Underarm & $21(100)$ & $8(100)$ \\
\hline Hands & $6(29)$ & $4(50)$ \\
\hline Feet & $6(29)$ & $3(37.5)$ \\
\hline Head & $6(29)$ & $5(62.5)$ \\
\hline Back & $4(19)$ & $5(62.5)$ \\
\hline Groin & $0(0)$ & $2(25)$ \\
\hline \multicolumn{3}{|l|}{ Sex, n (\%) } \\
\hline Male & $5(24)$ & $4(50)$ \\
\hline Female & $1676)$ & $4(50)$ \\
\hline Age (years), mean (range) & $36(18-57)$ & $13.1(10-17)$ \\
\hline \multicolumn{3}{|l|}{ Race, n (\%) } \\
\hline White & $16(76)$ & $3(37.5)$ \\
\hline Black & $1(5)$ & $0(0)$ \\
\hline Hispanic & $4(19)$ & $3(37.5)$ \\
\hline Other & $0(0)$ & $2(25.0)$ \\
\hline \multicolumn{3}{|c|}{$\begin{array}{l}\text { Current medication use for any }{ }^{\mathrm{b}} \text { excessive } \\
\text { sweating, } \mathrm{n}(\%)^{\mathrm{c}}\end{array}$} \\
\hline Over-the-counter & $12(57)$ & $7(87.5)$ \\
\hline Prescription & $10(48)$ & $1(12.5)$ \\
\hline \multicolumn{3}{|c|}{$\begin{array}{l}\text { Previous medication use for any }{ }^{b} \text { excessive } \\
\text { sweating, } \mathrm{n}(\%)^{c}\end{array}$} \\
\hline Over-the-counter & $20(95)$ & $7(87.5)$ \\
\hline Prescription & $16(76)$ & $1(12.5)$ \\
\hline
\end{tabular}

${ }^{a}$ Total sum exceeds $100 \%$, as participants could report excessive sweating in multiple locations

${ }^{\mathrm{b}}$ Excessive sweating refers to any area of excessive sweating (ie, not restricted to axillary sweating)

c Total sum exceeds $100 \%$, as participants could report concurrent use of more than one prescription and/or over-the-counter medication
Minor modifications to item wording were incorporated as needed following each round of interviews to ensure content was clear, comprehensive, and easy to understand. Concepts pertaining to underarm odor severity and interference with sleep were not generally endorsed by patients and deleted, as patients indicated that it was the anxiety about having odor that was most impactful and not necessarily the odor itself, and that hyperhidrosis symptoms typically occurred during the day and not while sleeping. The PGIC was also tested, with no revisions made in all three rounds of interviews as patients found the item clear and easy to answer in the context of receiving hyperhidrosis treatments. No new concepts were introduced as part of the concept elicitation portion of the interviews, and concept saturation was reached across the three sets of qualitative interviews. Thus, no additional patient interviews were required for further input.

The level of bother was consistent across both adult and pediatric samples. The most commonly reported impacts of hyperhidrosis in both populations included feeling embarrassed/self-conscious, modifying types of clothes worn, concern about underarm odor, changing shirts throughout the day, anxiety about sweating, ruined clothes, and physically uncomfortable due to excessive underarm wetness. Adults reported work-related impairments such as limiting interactions with work colleagues and nonmandatory work activities, consistent with the school-related impairments such as avoiding schoolbased activities and difficulty studying reported by the pediatric sample.

Like the adult interviewees, pediatric interviewees $(N=8)$ reported being bothered or burdened by the following: feeling embarrassed; sweating a lot more than others in similar circumstances; feeling sweat dripping down sides of the body; needing to cover up their sweating with hair or jackets/sweatshirts, even in hot weather;

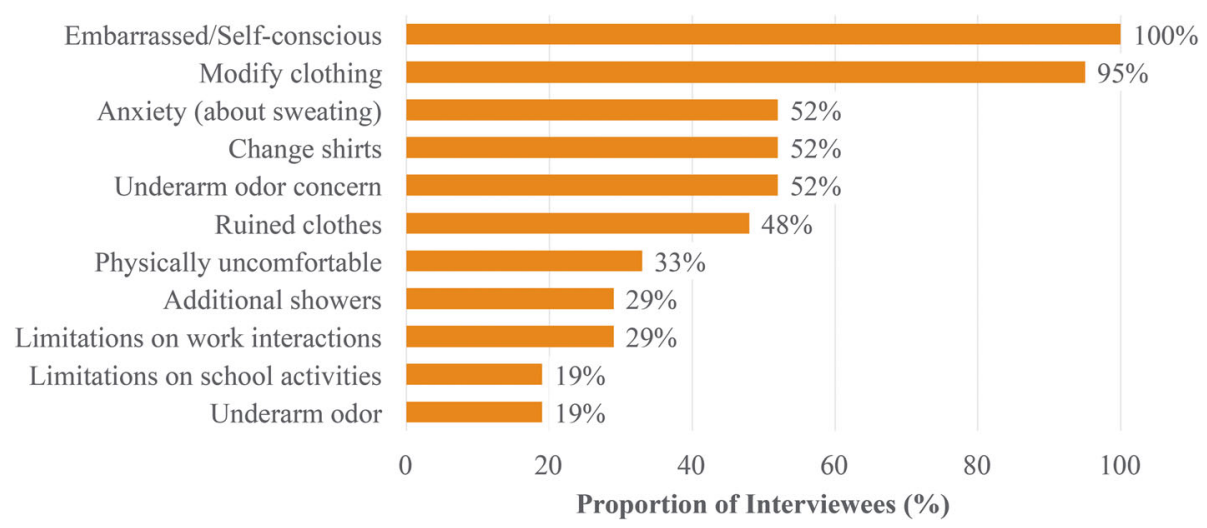

Fig. 2 Areas of bother and impact associated with axillary hyperhidrosis (Adult Patient Interviews; $N=21$ ) 
needing to "air their armpits out"; feeling like they have a bad smell; feeling sticky; not liking the wet feeling; and feeling itchy. Of note, adults did not report feeling itchy in conjunction with their excessive sweating. The three 17-year-old participants reported that their excessive underarm sweating began between during 6th or 8th grade. Three other participants (aged 10, 11, and 13 years) reported that their excessive sweating began around 4th grade and was most noticeable during sports or other physical activities. The remaining participants (aged 10 and 11 years) did not know when their excessive sweating began, stating that it was likely around age 4 to 5 years.

All 17-year-olds $(N=3)$ found the instructions and recall period on the ASDD clear and easy to understand. In contrast, 4 of the 5 younger participants (aged 10 to 13 years) had difficulty reading and understanding the instructions and/or understanding the $24-\mathrm{h}$ recall period. Based on these findings, the instructions and recall periods used in the ASDD-C were modified for patients $<16$ years of age, as reflected in Table 1 . Many of the younger pediatric interviewees had difficulty understanding and answering the ASDD items addressing the impact and burden of sweating (Items 3 and 4, respectively) even after modification; therefore, these items were eliminated from the ASDD-C. As many also had difficulty with the extended recall periods for the Weekly Impact items and the PGIC, these items were not administered to patients $<16$ years of age in the phase 3 trials.

The PRO measures resulting from the qualitative phase of research are shown in Table 1 . Item 1 is a gating question followed by Item 2, which queries level of axillary sweating severity using a 0 -to-10 numeric rating scale. The remaining two ASDD items are only administered to those 16 years of age and older and address impact and bothersomeness associated with axillary sweating (Items 3 and 4, respectively). Concurrent with the development of the ASDD, additional supportive assessments were included for use in patients $\geq 16$ years of age as follows: six items to assess the impact of hyperhidrosis on a weekly basis (Weekly Impact items; Table 1) and the single-item PGIC to assess overall change in hyperhidrosis severity over the study course (Table 1). As described above, a child-specific, 2-item version of the ASDD (ASDD-C; Table 1) was similarly developed for use in patients $\geq 9$ to $<16$ years of age.

\section{Psychometric evaluation}

The phase 2 and phase 3 patient pools were similar (Table 3) with the exception that the phase 3 trials allowed for the inclusion of younger patients. Specifically, 32 patients that were $\geq 9$ to $<16$ years of age (average age 13.8 years) were included in the phase 3 studies and administered the 2-item ASDD-C.

\section{Descriptive statistics and reliability}

No floor/ceiling effects were identified for the ASDD/ ASDD-C axillary sweating severity (Item 2 ) and impact (Item 3) items; there was some ceiling effect (21.4\%) for the bothersomeness item (Item 4; Table 4), which indicated that approximately one fifth of patients were extremely bothered by their condition at baseline. The ASDD also demonstrated adequate test-retest reliability

Table 3 Patient Demographic and Hyperhidrosis History Data From Phase 2 and Phase 3 Trials

\begin{tabular}{|c|c|c|c|c|}
\hline \multirow[t]{2}{*}{ Characteristic } & \multicolumn{2}{|c|}{$\begin{array}{l}\text { Phase 2: } \\
\text { DRM04-HH02 }(N=105)\end{array}$} & \multicolumn{2}{|l|}{$\begin{array}{l}\text { Phase } 3 \text { Pooled: } \\
\text { ATMOS-1 \& ATMOS-2 }\end{array}$} \\
\hline & & & Age $-9-15$ Years $(N=32)$ & $\begin{array}{l}\text { Age } \geq 16 \text { Years } \\
(N=665)\end{array}$ \\
\hline Age (years), mean $\pm S D$ & $33.3 \pm 11.7$ & $32.7 \pm 11.4$ & $13.8 \pm 1.37$ & $33.6 \pm 10.9$ \\
\hline Axillary hyperhidrosis history (years), mean \pm SD & NA & $15.5 \pm 10.8$ & $3.4 \pm 2.77$ & $16.1 \pm 10.7$ \\
\hline Female, n (\%) & $48(45.7)$ & $371(53.2)$ & $27(84.4 \%)$ & $344(51.7)$ \\
\hline White, $n(\%)$ & $91(86.7)$ & $570(81.8)$ & $26(81.3 \%)$ & $544(81.8)$ \\
\hline \multicolumn{5}{|c|}{ Weekly Impact Items, n (\%) responding Yes to each statement } \\
\hline Change shirt during the day & \multicolumn{2}{|l|}{79 (75.2\%) } & N/A & $517(77.7 \%)$ \\
\hline Take more than 1 shower or bath during the day & \multicolumn{2}{|l|}{$58(55.2 \%)$} & N/A & $345(51.9 \%)$ \\
\hline Feel less confident & \multicolumn{2}{|l|}{$84(80.0 \%)$} & N/A & $551(82.9 \%)$ \\
\hline Feel embarrassed & \multicolumn{2}{|l|}{$88(83.8 \%)$} & N/A & $583(87.7 \%)$ \\
\hline Avoid interactions with others & \multicolumn{2}{|l|}{$51(48.6 \%)$} & N/A & $402(60.5 \%)$ \\
\hline Prevent from doing activities & \multicolumn{2}{|l|}{$43(41.0 \%)$} & N/A & $352(52.9 \%)$ \\
\hline Weekly Impact Summary, ${ }^{a}$ mean \pm SD & \multicolumn{2}{|l|}{$3.8 \pm 1.90$} & N/A & $4.1 \pm 1.90$ \\
\hline
\end{tabular}

N/A Not available, data not collected during the study course, SD Standard deviation

aSummary score is derived by assigning a score of 1 for each "Yes" response (range 0 to 6). Validity of the Weekly Impact Summary score has not been tested via dimensionality analyses and should be considered exploratory at this stage 
Table 4 ASDD/ASDD-C Measurement Properties Evaluated in Phase 2 and Phase 3 Trials

\begin{tabular}{|c|c|c|c|c|c|}
\hline \multirow[t]{2}{*}{ Measurement Property } & & & \multirow{2}{*}{$\begin{array}{l}\text { Phase 2: } \\
\text { DRM04-HH02 } \\
(\mathrm{N}=105)\end{array}$} & \multicolumn{2}{|c|}{$\begin{array}{l}\text { Phase } 3 \text { Pool: } \\
\text { ATMOS-1 \& ATMOS-2 }\end{array}$} \\
\hline & & & & $\begin{array}{l}\text { Age } 9-15 \text { Years } \\
(N=32)\end{array}$ & $\begin{array}{l}\text { Age } \geq 16 \text { Years } \\
(N=665)\end{array}$ \\
\hline \multirow{9}{*}{$\begin{array}{l}\text { Mean } \pm \text { SD [median] } \\
\text { Floor and Ceiling Effects, } \\
\text { Non-response Bias, N(\%) }\end{array}$} & \multirow[t]{3}{*}{ Axillary Sweating Severity (Item 2) } & Baseline $^{a}$ & $6.8 \pm 1.9[7.0]$ & $6.8 \pm 2.5[7.0]$ & $7.2 \pm 1.6[7.4]$ \\
\hline & & Minimum (0) & $3(2.9 \%)$ & $0(0 \%)$ & $1(0.2 \%)$ \\
\hline & & Maximum (10) & $9(8.6 \%)$ & $6(18.8 \%)$ & $83(12.5 \%)$ \\
\hline & \multirow[t]{3}{*}{ Impact (Item 3) } & Baseline $^{a}$ & $2.2 \pm 0.9[2.2]$ & \multirow[t]{3}{*}{ N/A } & $2.4 \pm 0.9[2.3]$ \\
\hline & & Minimum (0) & $12(11.4 \%)$ & & $31(4.7 \%)$ \\
\hline & & Maximum (4) & $10(9.5 \%)$ & & $105(15.8 \%)$ \\
\hline & \multirow[t]{3}{*}{ Bothersomeness (Item 4) } & Baseline $^{a}$ & $2.3 \pm 0.9[2.3]$ & \multirow[t]{3}{*}{ N/A } & $2.6 \pm 0.9[2.6]$ \\
\hline & & Minimum (0) & $11(10.5 \%)$ & & $13(2.0 \%)$ \\
\hline & & Maximum (4) & $15(14.3 \%)$ & & $145(21.8 \%)$ \\
\hline \multirow[t]{3}{*}{ Test-Retest Reliability, ICC } & Axillary Sweating Severity (Item 2) & $\begin{array}{l}\text { Week } 4 \text { - } \\
\text { Week } 3\end{array}$ & 0.91 & 0.92 & 0.94 \\
\hline & Impact (Item 3) & $\begin{array}{l}\text { Week } 4 \text { - } \\
\text { Week } 3\end{array}$ & 0.89 & N/A & 0.90 \\
\hline & Bothersomeness (Item 4) & $\begin{array}{l}\text { Week } 4 \text { - } \\
\text { Week } 3\end{array}$ & 0.88 & N/A & 0.89 \\
\hline Item 2 Responsiveness & & $\begin{array}{l}\text { Week } 4 \text { - } \\
\text { Baseline }\end{array}$ & & & \\
\hline Sweat Production, ${ }^{b} r^{c}$ & & & $0.21^{f}$ & $0.01^{\mathrm{d}, \mathrm{e}}$ & $0.22^{\mathrm{d}, e}$ \\
\hline Effect Size of Change (SD Baseline units) & & & -2.2 & -2.0 & -2.4 \\
\hline Standardized Response Mean & & & -1.6 & -0.70 & -1.5 \\
\hline
\end{tabular}

ASDD Axillary Sweating Daily Diary, ICC Intraclass correlation, SD Standard deviation

${ }^{a}$ For subjects with Week 4 scores

${ }^{\mathrm{b}}$ Measured gravimetrically

' Based on the change from Baseline to Week 4 in ASDD Item 2 scores and sweat production

${ }^{\mathrm{d}}$ Spearman correlation coefficient

e Based on the change from Baseline to Week 4 in ASDD Item 2 scores and the natural logarithm of sweat production

${ }^{\mathrm{f}}$ Pearson correlation coefficient

between Week 3 and 4 (ICCs $\sim 0.9$ for Items $2-4$ ), well above the 0.70 threshold.

\section{Construct validity}

The pattern of correlations between ASDD items and other efficacy measures is supportive of the construct validity of the ASDD axillary sweating severity item (Item 2; Table 5). Specifically, strong positive correlations $(r \geq 0.5)$ were observed between ASDD Item 2 and HDSS grade at Week 4, as well as between the changes in both measures from Baseline. As expected, ASDD Item 2 was moderately to strongly correlated with the DLQI/C-DLQI $(r=0.61 / 0.51$ at Week 4 and $r=0.63$ / 0.51 between changes in both measures from Baseline). Correlations between ASDD Item 2 and sweat production were weak to moderate at Week $4(r=0.17$ to 0.33$)$ and were weak for the changes in both measures from Baseline ( $r=0.17$ to 0.18$)$, which was expected given the differences in reference period for these measures (ie, ASDD Item 2 daily assessments were averaged over a 7-day period whereas sweat production was assessed at a single point in time, once each week). Similar trends were noted for both the adult and pediatric populations in the phase 3 study. Exploratory analyses to evaluate the relationship between all newly developed items (ASDD, Weekly Impact, PGIC) showed strong relationships across the different measures. ASDD inter-item correlations were all $>0.80$. While severity, impact, and bothersomeness in the ASDD may represent different constructs of hyperhidrosis, they are likely to be strongly associated with one another. Furthermore, ASDD Item 2 was strongly correlated with the Weekly Impact summary. In addition, the correlation between the change from Baseline in ASDD Item 2 was strongly correlated with change from Baseline in the Weekly Impact summary and the PGIC at Week 4 (Table 5). Finally, ASDD items addressing the impact and burden of sweating (Items 3 and 4 , respectively) demonstrated a similar pattern of correlations (strong correlations with HDSS and Weekly Impact summary, moderate to strong correlation with DLQI, and a weak correlation with sweat production), though results 
Table 5 ASDD Construct Validity Correlations at Week 4 (Phase 2 data; N=105 or Phase 3 data; $N=697$ )

\begin{tabular}{|c|c|c|c|c|c|c|}
\hline ASDD Item & & $\begin{array}{l}\text { HDSS Grade, } \\
\text { Pearson } \\
\text { correlation }\end{array}$ & $\begin{array}{l}\text { Sweat Production, }{ }^{a} \\
\text { Pearson correlation }\end{array}$ & $\begin{array}{l}\text { DLQI/C-DLQI, } \\
\text { Spearman } \\
\text { correlation }\end{array}$ & $\begin{array}{l}\text { Weekly Impact } \\
\text { Summary, Pearson } \\
\text { correlation }\end{array}$ & $\begin{array}{l}\text { PGIC, } \\
\text { Spearman } \\
\text { correlation }\end{array}$ \\
\hline \multicolumn{7}{|l|}{ Week 4 Average } \\
\hline \multirow[t]{3}{*}{ Axillary Sweating Severity (Item 2) } & Phase 2 & $0.73^{*}$ & $0.33^{*}$ & N/A & $0.52^{*}$ & $0.62^{*}$ \\
\hline & $\begin{array}{l}\text { Phase } 3 \\
9-15 y o\end{array}$ & $0.71^{*}$ & 0.17 & $0.51^{*}$ & N/A & N/A \\
\hline & $\begin{array}{l}\text { Phase } \\
3 \geq \\
16 \text { yo }\end{array}$ & $0.70^{*}$ & $0.18^{*}$ & $0.61^{*}$ & $0.66^{*}$ & $0.70^{*}$ \\
\hline \multirow[t]{2}{*}{ Impact (Item 3) } & Phase 2 & $0.71^{*}$ & $0.24^{*}$ & N/A & $0.62^{*}$ & $0.65^{* \mathrm{~b}}$ \\
\hline & $\begin{array}{l}\text { Phase } 3 \\
\text { Pooled }\end{array}$ & $0.72^{*}$ & $0.13^{*}$ & $0.65^{*}$ & $0.68^{*}$ & $0.65^{*}$ \\
\hline \multirow[t]{2}{*}{ Bothersomeness (Item 4) } & Phase 2 & $0.79^{*}$ & $0.27^{*}$ & - & $0.64^{*}$ & $0.70^{* b}$ \\
\hline & $\begin{array}{l}\text { Phase } 3 \\
\text { Pooled }\end{array}$ & $0.74^{*}$ & $0.13^{*}$ & $0.66^{*}$ & $0.69^{*}$ & $0.66^{*}$ \\
\hline \multicolumn{7}{|l|}{ Change from Baseline to Week 4} \\
\hline \multirow[t]{3}{*}{ Axillary Sweating Severity (Item 2) } & Phase 2 & $0.57^{*}$ & 0.17 & N/A & $0.65^{*}$ & $0.48^{*}$ \\
\hline & $\begin{array}{l}\text { Phase } 3 \\
9-15 y o\end{array}$ & $0.71^{*}$ & 0.17 & $0.51^{*}$ & N/A & N/A \\
\hline & $\begin{array}{l}\text { Phase } \\
3 \geq \\
16 \text { yo }\end{array}$ & $0.70^{*}$ & 0.18 & $0.63^{*}$ & $0.62^{*}$ & $0.68^{*}$ \\
\hline \multirow[t]{2}{*}{ Impact (Item 3) } & Phase 2 & $0.51^{*}$ & 0.05 & N/A & $0.57^{*}$ & $0.27^{*}$ \\
\hline & $\begin{array}{l}\text { Phase } 3 \\
\text { Pooled }\end{array}$ & $0.64^{*}$ & 0.01 & $0.51^{*}$ & $0.65^{*}$ & $0.57^{*}$ \\
\hline \multirow[t]{2}{*}{ Bothersomeness (Item 4) } & Phase 2 & $0.58^{*}$ & 0.13 & N/A & $0.61^{*}$ & $0.31^{*}$ \\
\hline & $\begin{array}{l}\text { Phase } 3 \\
\text { Pooled }\end{array}$ & $0.65^{*}$ & 0.02 & $0.53^{*}$ & $0.65^{*}$ & $0.59^{*}$ \\
\hline
\end{tabular}

ASDD Axillary Sweating Daily Diary, HDSS Hyperhidrosis Disease Severity Scale, PG/C Patient Global Impression of Change

${ }^{*} p \leq 0.05$

${ }^{a}$ Measured gravimetrically

${ }^{\mathrm{b}}$ Weekly average for PGIC is not applicable

Entries marked N/A indicate that the assessment was not performed for that study and/or population

were mixed with respect to Items 3 and 4 correlation with PGIC for the phase 2 and phase 3 data sets, with stronger correlations observed in the phase 3 set (Table 5).

\section{Known-groups validity}

Statistically significant differences in mean ASDD Item 2 scores and HDSS grade strata $(p<0.0001)$, gravimetricallymeasured sweat production quartile $(p=0.009$ for phase 2; $p<0.001$ for phase 3$)$ at Week 4 , and DLQI $(p<0.0001)$ (Table 6) support the known-groups validity of ASDD Item 2 in the adult population. Similar trends were noted in the phase 3 pediatric population for HDSS Scores. While ASDD Item 2 scores were higher (more severe patientreported sweating) for patients with greater sweat production (4.1 vs 6.3 ), the difference was not statistically significant, which could in part be due to the smaller sample size in the pediatric population $(n=32)$. Only 1 pediatric patient reported a very large to extremely large HRQOL impact due their skin condition on the
C-DLQI; this patient reported a mean weekly ASDD score of 9.8 compared to 4.4 in the group with minimal HRQOL impact. Although no specific predictions were preplanned for ASDD Items 3 (impact) and 4 (bother), data in Table 6 show that these items were also able to discriminate between known groups.

\section{Responsiveness}

The responsiveness of ASDD/ASDD-C Item 2 (ability to detect change in sweating severity) was demonstrated by large effect sizes (ranging from -2.2 to -2.4 ), as well as by correlations that were within the expected range for the change in Item 2 and the change in the gravimetric measures of sweat production (Table 4). In addition, the correlation between the change from Baseline in ASDD Item 2 was strongly correlated with change from Baseline in the Weekly Impact summary and the PGIC at Week 4 (Table 5). 
Table 6 Known-Groups Validity - ASDD by HDSS, Gravimetry, and DLQI/CDLQI severity thresholds at Week 4

\begin{tabular}{|c|c|c|c|c|}
\hline & & \multirow{2}{*}{$\begin{array}{l}\text { Phase 2: } \\
\text { DRM04-HH02 } \\
(N=105)\end{array}$} & \multicolumn{2}{|c|}{ Phase 3 Pooled: ATMOS-1 \& ATMOS-2 } \\
\hline & & & $\begin{array}{l}\text { Age } 9-15 \text { Years } \\
(N=32)\end{array}$ & $\begin{array}{l}\text { Age } \geq 16 \text { Years } \\
(N=665)\end{array}$ \\
\hline \multirow[t]{12}{*}{ ASDD Item 2 (Week 4 Average) } & HDSS, mean (SD) & & & \\
\hline & Grade 1 & $0.9 \pm 1.2(n=35)$ & $2.0 \pm 0.6(n=5)$ & $1.1 \pm 1.6(n=208)$ \\
\hline & Grade $\geq 3$ & $5.3 \pm 1.4(n=14)$ & $6.8 \pm 1.86(n=13)$ & $6.5 \pm 2.1(n=154)$ \\
\hline & $p$-value & $<0.001$ & $<0.001$ & $<0.001$ \\
\hline & Sweat Production, ${ }^{a}$ mean (SD) & & & \\
\hline & 1st Quartile & $1.8 \pm 1.7(n=19)$ & $4.1 \pm 3.2(n=3)$ & $2.6 \pm 2.6(n=166)$ \\
\hline & 4th Quartile & $3.4 \pm 2.0(n=20)$ & $6.3 \pm 1.9(n=15)$ & $4.2 \pm 2.8(n=168)$ \\
\hline & $p$-value & 0.009 & 0.125 & $<0.001$ \\
\hline & DLQI/C-DLQI, mean (SD) & & & \\
\hline & No to moderate impact & N/A & $4.4 \pm 2.5(n=27)$ & $2.9 \pm 2.5(n=571)$ \\
\hline & Very large to extremely large impact & N/A & $9.8(n=1)$ & $7.3 \pm 2.4(n=7)$ \\
\hline & $p$-value & N/A & $N / A^{b}$ & $<0.001$ \\
\hline \multirow[t]{12}{*}{ ASDD Item 3 (Week 4 Average) } & HDSS, mean (SD) & & & \\
\hline & Grade 1 & $0.2 \pm 0.4(n=35)$ & N/A & $0.2 \pm 0.3(n=182)$ \\
\hline & Grade $\geq 3$ & $1.5 \pm 0.7(n=14)$ & N/A & $2.1 \pm 1.0(n=114)$ \\
\hline & $p$-value & $<0.001$ & N/A & $<0.001$ \\
\hline & Sweat Production, ${ }^{a}$ mean (SD) & & & \\
\hline & 1st Quartile & $0.5 \pm 0.6(n=19)$ & N/A & $0.7 \pm 0.8(n=146)$ \\
\hline & 4th Quartile & $1.0 \pm 0.7(n=20)$ & N/A & $1.2 \pm 1.0(n=134)$ \\
\hline & $p$-value & 0.026 & N/A & $<0.001$ \\
\hline & DLQI/C-DLQI, mean (SD) & & & \\
\hline & No to moderate impact & N/A & N/A & $0.8 \pm 0.9(n=508)$ \\
\hline & Very large to extremely large impact & N/A & N/A & $3.0 \pm 1.0(n=5)$ \\
\hline & $p$-value & N/A & N/A & $<0.001$ \\
\hline \multirow[t]{12}{*}{ ASDD Item 4 (Week 4 Average) } & HDSS, mean (SD) & & & \\
\hline & Grade 1 & $0.2 \pm 0.3(n=35)$ & N/A & $0.2 \pm 0.3(n=182)$ \\
\hline & Grade $\geq 3$ & $1.7 \pm 0.7(n=14)$ & N/A & $2.3 \pm 1.0(n=114)$ \\
\hline & $p$-value & $<0.001$ & N/A & $<0.001$ \\
\hline & Sweat Production, ${ }^{a}$ mean (SD) & & & \\
\hline & 1st Quartile & $0.5 \pm 0.5(n=19)$ & N/A & $0.8 \pm 0.8(n=146)$ \\
\hline & 4th Quartile & $1.2 \pm 0.7(n=20)$ & N/A & $1.3 \pm 1.0(n=134)$ \\
\hline & $p$-value & 0.002 & N/A & $<0.001$ \\
\hline & DLQI/C-DLQI, mean (SD) & & & \\
\hline & No to moderate impact & N/A & N/A & $0.9 \pm 0.9(n=508)$ \\
\hline & Very large to extremely large impact & N/A & N/A & $3.0 \pm 1.1(n=5)$ \\
\hline & $p$-value & N/A & N/A & $<0.001$ \\
\hline
\end{tabular}

ASDD Axillary Sweating Daily Diary, HDSS Hyperhidrosis Disease Severity Scale, SD Standard deviation

${ }^{\text {a }}$ Measured gravimetrically; ${ }^{\mathrm{b}}$ Not calculable due to sample size $(N=1)$

$P$ value for two sample t-test

HDSS Grade $1=$ My sweating is never noticeable and never interferes with my daily activities; Grade $3=$ My sweating is barely tolerable and frequently interferes with my daily activities; Grade $4=$ My sweating is intolerable and always interferes with my daily activities

Quartiles for gravimetric measurement of sweat production at Week 4 defined as 25 th percentile $(8.7 \mathrm{mg} / \mathrm{axilla} / 5 \mathrm{~min})$ and $75 \mathrm{th}$ percentile $(34.95 \mathrm{mg} / \mathrm{axilla} / 5 \mathrm{~min})$ DLQI: no to moderate impact $=$ range $0-10$; large to extremely large impact $=$ range $21-30$; CDLQl: no to moderate impact $=$ range $0-12$; large to extremely large impact $=$ range $19-20$ 
Table 7 Responder Estimates for ASDD Axillary Sweating Severity Item (Item 2): Anchor-Based Method (Phase 2 Data)

\begin{tabular}{|c|c|c|c|c|c|c|c|c|}
\hline \multirow[t]{2}{*}{ PGIC Response } & \multicolumn{4}{|c|}{$\begin{array}{l}\text { Phase 2: DRM04-HH02 } \\
(N=105)\end{array}$} & \multicolumn{4}{|c|}{$\begin{array}{l}\text { Phase } 3 \text { Pooled: ATMOS-1 \& ATMOS-2 } \\
(N=697)\end{array}$} \\
\hline & n (\%) & $\begin{array}{l}\text { ASDD Axillary Sweating } \\
\text { Severity (Average Weekly } \\
\text { Change), } \\
\text { mean } \pm \text { SD [median] }\end{array}$ & n & $\begin{array}{l}\text { HDSS change score } \\
\text { (Average Weekly } \\
\text { Change), mean } \pm \text { SD } \\
\text { [median] }\end{array}$ & n (\%) & $\begin{array}{l}\text { ASDD Axillary Sweating } \\
\text { Severity (Average Weekly } \\
\text { Change), } \\
\text { mean } \pm \text { SD [median] }\end{array}$ & n (\%) & $\begin{array}{l}\text { HDSS change score } \\
\text { (Average Weekly } \\
\text { Change), mean } \pm \text { SD } \\
\text { [median] }\end{array}$ \\
\hline 1 = Much better & $\begin{array}{l}50 \\
(64.1)\end{array}$ & $-5.1 \pm 2.5[-5.5]$ & 45 & $-1.9 \pm 0.80[-2.0]$ & $\begin{array}{l}276 \\
(39.6)\end{array}$ & $-5.7 \pm 2.05[-5.7]$ & $\begin{array}{l}276 \\
(39.6)\end{array}$ & $-2.0 \pm 0.71[-2.0]$ \\
\hline $\begin{array}{l}2=\text { Moderately } \\
\text { better }\end{array}$ & $\begin{array}{l}11 \\
(14.1)\end{array}$ & $-3.8 \pm 2.7[-3.4]$ & 10 & $-1.5 \pm 0.71[-1.0]$ & $\begin{array}{l}111 \\
(15.9)\end{array}$ & $-3.8 \pm 2.28[-3.7]$ & $\begin{array}{l}111 \\
(15.9)\end{array}$ & $-1.4 \pm 0.66[-1.0]$ \\
\hline $3=\mathrm{A}$ little better & $\begin{array}{l}13 \\
(16.7)\end{array}$ & $-2.3 \pm 1.2[-1.9]$ & 14 & $-0.6 \pm 0.76[0.0]$ & $\begin{array}{l}119 \\
(17.1)\end{array}$ & $-2.3 \pm 1.97[-1.9]$ & $\begin{array}{l}119 \\
(17.1)\end{array}$ & $-0.9 \pm 0.71[-1.0]$ \\
\hline $4=$ No difference & $4(5.1)$ & $-1.7 \pm 0.7[-1.9]$ & 3 & $-0.3 \pm 0.58[0.0]$ & $\begin{array}{l}82 \\
(11.8)\end{array}$ & $-1.0 \pm 1.69[-0.6]$ & $\begin{array}{l}82 \\
(11.8)\end{array}$ & $-0.5 \pm 0.74[0.0]$ \\
\hline $5=\mathrm{A}$ little worse & 0 & - & 0 & - & $11(1.6)$ & $0.2 \pm 1.54[0.3]$ & $11(1.6)$ & $-0.4 \pm 0.67[0.0]$ \\
\hline $\begin{array}{l}6=\text { Moderately } \\
\text { worse }\end{array}$ & 0 & - & 0 & - & 0 & - & 0 & - \\
\hline $7=$ Much worse & 0 & - & 0 & - & 0 & - & 0 & - \\
\hline
\end{tabular}

Bold text represents the preferred threshold for meaningful response consistent with FDA guidance ("moderately better")

ASDD Axillary Sweating Daily Diary, PGIC Patient Global Impression of Change, SD Standard deviation

A PGIC rating of "moderately better" within this scale corresponded to a 3.8-point change on the ASDD Item 2 in both phase 2 and phase 3 studies (Table 7). A "moderately better" category was used to indicate a clinically meaningful change from the patient perspective. Patients achieving reductions in weekly average scores on ASDD Item 2 of $\geq 4$ points were defined as responders to treatment. Within-person changes in HDSS scores against PGIC responses followed a similar trend as that of ASDD; similarly, within-person change scores on ASDD calculated across different magnitudes of change in HDSS scores showed that as HDSS scores improved, so did ASDD scores (Tables 7 and 8).

\section{Discussion}

The purpose of this research was to develop the ASDD and ASDD-C and demonstrate validity of Item 2, intended to measure severity of axillary sweating among patients with axillary hyperhidrosis in both adults and pediatric patients aged 9 and above. Qualitative and quantitative analyses presented here demonstrate strong relationships with other established outcomes in hyperhidrosis (construct and known-groups validity), good reproducibility (reliability), and establishment of a threshold indicating a meaningful magnitude of response via anchor-based methods (responsiveness) for use in evaluating axillary hyperhidrosis treatments in interventional studies. Validation analyses were highly consistent between the phase 2 and phase 3 studies, providing increased confidence in ASDD measurement properties and particularly the responder definition. This research highlights the importance of capturing the patient perspective for this condition, particularly in light of the limitations associated with more objective methods of assessing sweat production.

Given the level of unmet need among hyperhidrosis patients, novel assessment tools are needed to support

Table 8 ASDD Change Scores Across HDSS Improvements

\begin{tabular}{|c|c|c|c|c|}
\hline \multirow[t]{2}{*}{ HDSS Score } & \multicolumn{2}{|c|}{$\begin{array}{l}\text { Phase 2: DRM04-HH02 } \\
(N=105)\end{array}$} & \multicolumn{2}{|c|}{$\begin{array}{l}\text { Phase } 3 \text { Pooled: ATMOS-1 \& ATMOS-2 } \\
(N=697)\end{array}$} \\
\hline & n (\%) & $\begin{array}{l}\text { ASDD Axillary Sweating Severity } \\
\text { (Average Weekly Change), mean } \pm \text { SD } \\
\text { [median] }\end{array}$ & n (\%) & $\begin{array}{l}\text { ASDD Axillary Sweating Severity } \\
\text { (Average Weekly Change), mean } \pm \text { SD } \\
\text { [median] }\end{array}$ \\
\hline 3-point improvement & $13(16.7 \%)$ & $-6.2 \pm 2.68[-6.6]$ & 74 (10.6) & $-6.7 \pm 2.29[-6.9]$ \\
\hline 2-point improvement & $29(37.2 \%)$ & $-5.2 \pm 2.10[-5.3]$ & $251(36)$ & $-5.2 \pm 2.13[-5.4]$ \\
\hline 1-point improvement & $24(30.8 \%)$ & $-3.3 \pm[-3.2]$ & $247(35.4)$ & $-2.9 \pm 2.17[-3.0]$ \\
\hline No improvement & $12(15.4 \%)$ & $-1.9 \pm 0.92[-2.0]$ & $113(16.2)$ & $-0.9 \pm 1.91[-0.5]$ \\
\hline 1-point worsening & 0 & - & $12(1.7)$ & $-0.5 \pm 1.47[-0.5]$ \\
\hline 2-point worsening & 0 & - & - & - \\
\hline 3-point worsening & 0 & - & - & - \\
\hline
\end{tabular}


future research and the development of new treatments. Although the HDSS has historically been used in the clinical trial setting to characterize patients with hyperhidrosis, it cannot be used in support of product approvals because patient input was not formally taken into consideration during its development. In addition, the HDSS combines two concepts within the same item (symptom severity and impact), limiting the ability to ascertain which of the two concepts has changed with treatment. The ASDD was developed in accordance with the recommendations outlined in the FDA PRO guidance to support U.S. product approvals [18]. This instrument was created based on patient input and clinical considerations, and the parallel development of a pediatric-specific version of the ASDD expands research capability across a range of patient populations. This novel PRO measure has added value in that ASDD/ ASDD-C axillary sweating severity item (Item 2) can support efficacy assessments for product approvals and labeling, which may facilitate approval of new treatments for axillary hyperhidrosis. In addition, the results of qualitative and quantitative analysis for impact (Item 3) and bothersomeness (Item 4) support their inclusion as key endpoints in clinical trials. The Weekly Impact items were developed based on patient input to explore impacts on various areas of patients' lives and offers valuable insight with regards to how patients cope with this condition on a regular basis. Additional research focusing on more distal impacts of the disease is warranted to further refine these items.

Several study limitations should be recognized. Foremost, the development of the ASDD/ASDD-C was based on a small, homogeneous patient population, which may not have reflected different levels of disease severity; additionally, the pediatric patients did not have a physician-confirmed diagnosis of hyperhidrosis for the qualitative development. Patients included in the phase 2 and phase 3 trials were also relatively homogeneous (ie, similar disease severity and demographic characteristics), potentially limiting generalizability of the measure. In addition, though the axillary sweating severity item is equivalent between the ASDD and ASDD-C, the cohort of patients $\geq 9$ and $<16$ years in the phase 3 trials was small. Despite this limitation, ASDD-C data in the pediatric population demonstrate results broadly consistent with those of adults. The focus of this research was primarily on the ASDD Item 2; while exploratory analyses showed that all the newly developed items demonstrated consistency with one another, dimensionality was not formally assessed to better understand their relationship with one another and whether it would be appropriate to derive summary scores. It also should be noted that initial draft items pertaining to severity were developed before patient engagement, which as a matter of process may be disadvantageous when seeking to identify constructs directly germane to a patient; however, for this hyperhidrosis measure, the concept of sweating severity was the focus of the study (Item 2) and was considered to be both straightforward and unambiguously relevant.

\section{Conclusions}

Taken together, the rigorous development and validation of ASDD/ASDD-C axillary sweating severity item support its use in the evaluation of axillary hyperhidrosis treatment in clinical trials. Strong qualitative and quantitative evidence also supports the use of the impact and bothersomeness items as key trial endpoints in adult trials. Further evaluation of this measure in future research studies is warranted to demonstrate consistent performance across different axillary hyperhidrosis populations and in different study contexts.

Familiarity with the ASDD/ASDD-C along with the Weekly Impact items among clinicians who treat patients with hyperhidrosis may facilitate a better understanding of the impact of this condition on patients' lives.

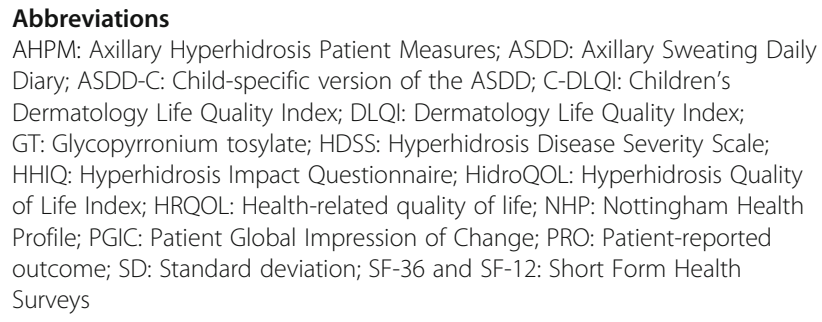

\section{Acknowledgements}

Medical writing support was provided by Prescott Medical Communications Group (Chicago, IL) with financial support from Dermira, Inc.

\section{Authors' contributions}

LN, DD, and SF: Methodology, data generation and analysis, manuscript editing and approval. DMP, AAH, DAG: Methodology, analysis, manuscript editing and approval. HH, JD, KKG and DI: Concept, analysis, manuscript editing and approval. All authors read and approved the final manuscript.

\section{Funding}

These studies were funded by Dermira, Inc. RTI-HS conducted the research described in this manuscript.

\section{Availability of data and materials}

The data that support the findings of this study are available from Dermira, Inc., but restrictions apply to the availability of these data, which were used under license for the current study, and so are not publicly available. Data are however available from the authors upon reasonable request and with permission of Dermira.

\section{Ethics approval and consent to participate}

Source data were from two phase 3 clinical trials that were reviewed and approved by local institutional review boards and independent ethics committees on May 13, 2015.

Consent for publication

Not applicable. 


\section{Competing interests}

LN, DD, and SF: Employee of RTI Health Solutions. DMP: Consultant (honoraria): Brickell Biotech, Inc.; Biofrontera AG; Celgene; Dermira, Inc.; DUSA Pharmaceuticals, Inc.; LEO Pharma; Novartis; Promius Pharma, LLC; Regeneron Pharmaceuticals, Inc.; Sanofi; TheraVida, Inc.; Valeant Pharmaceuticals International, Inc. Advisory board (honoraria): Pfizer, Inc. Investigator (grants/ research funding): Abbott Laboratories; Amgen, Inc;; Brickell Biotech, Inc.; Celgene; Dermavant Sciences; Eli Lilly and Company; LEO Pharma; Merck \& Co, Inc.; Novartis; Novo Nordisk A/S; Ortho Dermatologics; Peplin, Inc.; Photocure ASA; Promius Pharma, LLC; Regeneron Pharmaceuticals, Inc.; Stiefel Laboratories; Valeant Pharmaceuticals International, Inc. Investigator (honoraria): LEO Pharma; Pfizer, Inc. DAG: Consultant and investigator: Dermira, Inc. AAH: Investigator (research funding): Dermira, Inc. (paid to the UTHealth McGovern Medical School, Houston). Advisory board (honoraria): Dermira, Inc. Research funding (all monies paid to the UTHealth McGovern Medical School, Houston): Allergan, Plc;; Amgen, Inc.; Cassiopea; Celgene; Dermavant Sciences; Eli Lilly and Company; Galderma S.A.; GSK, Plc.; LEO Pharma; Mayne Pharma; Medimetriks Pharmaceuticals; Novan, Inc.; Promius Pharma, LLC; Vanda Pharmaceuticals. Honoraria: Amgen; GSK, PIc.; Pfizer, Inc.; Valeant Pharmaceutics International, Inc. HH, JD, KKG, and DI: Employee and stockholder of Dermira, Inc.

\section{Author details}

1RTI Health Solutions, 200 Park Offices Drive, Research Triangle Park, NC 27709, USA. ${ }^{2}$ Eastern Virginia Medical School Department of Dermatology and Virginia Clinical Research, Inc, 6160 Kempsville Road Suite 200A, Norfolk, VA 23452, USA. ${ }^{3}$ Department of Dermatology, Saint Louis University, 1755 S. Grand Blvd, St. Louis, MO 63104, USA. ${ }^{4}$ Department of Dermatology, UTHealth McGovern Medical School at Houston, 6655 Travis, Suite 980, Houston, TX 77030, USA. ${ }^{5}$ Dermira, Inc, 275 Middlefield Road, Suite 150, Menlo Park, CA CA 94025, USA.

\section{Received: 3 August 2018 Accepted: 15 August 2019} Published online: 05 September 2019

\section{References}

1. Grabell, D. A., \& Hebert, A. A. (2017). Current and emerging medical therapies for primary hyperhidrosis. Dermatology Therapy (Heidelb), 7, 25-36.

2. Doolittle, J., Walker, P., Mills, T., \& Thurston, J. (2016). Hyperhidrosis: An update on prevalence and severity in the United States. Archives of Dermatological Research, 308, 743-749.

3. Hamm, H. (2014). Impact of hyperhidrosis on quality of life and its assessment. Dermatologic Clinics, 32, 467-476.

4. Ambrogi, V., Campione, E., Mineo, D., Paterno, E. J., Pompeo, E., \& Mineo, T. C. (2009). Bilateral thoracoscopic T2 to T3 sympathectomy versus botulinum injection in palmar hyperhidrosis. The Annals of Thoracic Surgery, 88, 238-245.

5. Tetteh, H. A., Groth, S. S., Kast, T., Whitson, B. A., Radosevich, D. M., Klopp, A. C., D'Cunha, J., Maddaus, M. A., \& Andrade, R. S. (2009). Primary palmoplantar hyperhidrosis and thoracoscopic sympathectomy: A new objective assessment method. The Annals of Thoracic Surgery, 87, 267-274 discussion 274-265.

6. Patrick, D. L., \& Deyo, R. A. (1989). Generic and disease-specific measures in assessing health status and quality of life. Medical Care, 27, S217-S232.

7. Hamm, H., Naumann, M. K., Kowalski, J. W., Kutt, S., Kozma, C., \& Teale, C. (2006). Primary focal hyperhidrosis: Disease characteristics and functional impairment. Dermatology, 212, 343-353.

8. Weber, A., Heger, S., Sinkgraven, R., Heckmann, M., Elsner, P., \& Rzany, B. (2005). Psychosocial aspects of patients with focal hyperhidrosis. Marked reduction of social phobia, anxiety and depression and increased quality of life after treatment with botulinum toxin a. The British Journal of Dermatology, 152, 342-345.

9. Finlay, A. Y., \& Khan, G. K. (1994). Dermatology life quality index (DLQI)--a simple practical measure for routine clinical use. Clinical and Experimental Dermatology, 19, 210-216.

10. Kowalski, J. W., Eadie, N., Dagget, S., \& Lai, P.-Y. (2004). Validity and reliability of the hyperhidrosis disease severity scale (HDSS). Journal of the American Academy of Dermatology, 50

11. Solish, N., Bertucci, V., Dansereau, A., Hong, H. C., Lynde, C., Lupin, M., Smith, K. C., \& Storwick, G. (2007). A comprehensive approach to the recognition, diagnosis, and severity-based treatment of focal hyperhidrosis:
Recommendations of the Canadian hyperhidrosis advisory committee. Dermatologic Surgery, 33, 908-923.

12. Naumann, M. K., Hamm, H., \& Lowe, N. J. (2002). Effect of botulinum toxin type a on quality of life measures in patients with excessive axillary sweating: A randomized controlled trial. The British Journal of Dermatology, 147, 1218-1226.

13. Amir, M., Arish, A., Weinstein, Y., Pfeffer, M., \& Levy, Y. (2000). Impairment in quality of life among patients seeking surgery for hyperhidrosis (excessive sweating): Preliminary results. The Israel Journal of Psychiatry and Related Sciences, 37, 25-31.

14. de Campos, J. R., Kauffman, P., Werebe Ede, C., Andrade Filho, L. O., Kusniek, S., Wolosker, N., \& Jatene, F. B. (2003). Quality of life, before and after thoracic sympathectomy: Report on 378 operated patients. The Annals of Thoracic Surgery, 76, 886-891

15. Keller, S., Sekons, D., Scher, H., et al. (2001). A novel scale for assessing quality of life following bilateral endoscopic thoracic sympathectomy for palmar and plantar hyperhidrosis. In Abstract book of the 4th international symposium on sympathetic surgery (pp. Abstract OAbstract22) Abstract O-22.

16. Kamudoni, P., Mueller, B., \& Salek, M. S. (2015). The development and validation of a disease-specific quality of life measure in hyperhidrosis: The hyperhidrosis quality of life index (HidroQOL(c)). Quality of Life Research, 24, 1017-1027.

17. Kamudoni, P., Salek, M. S., Mueller, B., \& Mueller, C. (2012). Hyperhidrosis quality of life index (Hidroqol (c)): A novel patient reported outcome measure in hyperhidrosis. Journal of Investigative Dermatology, 132(S2), S73-S73.

18. Guidance for Industry. (2009). Patient-reported outcome measures: Use in medical product development to support labeling claims. In US Food \& Drug Administration (FDA).

19. Comparator study of the effect of glycopyrrolate and glycopyrronium in subjects with axillary hyperhidrosis (DRM04-HH02). https://clinicaltrials.gov/ ct2/show/study/NCT02129660?term=drm04\&cond=Hyperhidrosis\&rank=4. Accessed 12 Apr 2019

20. Glaser, D. A., Hebert, A. A., Nast, A., Werschler, W. P., Green, L., Mamelok, R. Drew, J., Quiring, J., \& Pariser, D. M. (2019). Topical Glycopyrronium Tosylate for the treatment of primary axillary hyperhidrosis: Results from the ATMOS1 and ATMOS-2 phase 3 randomized controlled trials. Journal of the American Academy of Dermatology, 80, 128-138.

21. Lewis-Jones, M. S., \& Finlay, A. Y. (1995). The Children's dermatology life quality index (CDLQI): Initial validation and practical use. The British Journal of Dermatology, 132, 942-949.

22. Nunnally, J. C., \& Bernstein, I. (1994). Psychometric theory. New York: McGrawHill.

23. Patient Global Impression of Change (PGIC). National Institute of Mental Health (NIMH) https://eprovide.mapi-trust.org/instruments/patient-globalimpression-of-change. Accessed 12 Apr 2019.

24. Cohen, J. (1992). A power primer. Psychological Bulletin, 112, 155-159.

25. Brandt, M., Bielfeldt, S., Springmann, G., \& Wilhelm, K. P. (2008). Influence of climatic conditions on antiperspirant efficacy determined at different test areas. Skin Research and Technology, 14, 213-219.

26. Hongbo, Y., Thomas, C. L., Harrison, M. A., Salek, M. S., \& Finlay, A. Y. (2005). Translating the science of quality of life into practice: What do dermatology life quality index scores mean? The Journal of Investigative Dermatology, 125, 659-664.

27. Waters, A., Sandhu, D., Beattie, P. E., Ezughah, F., \& Lewis-Jones, S. (2010). Severity stratification of Children's dermatology life quality index (CDLQI) scores. The British Journal of Dermatology, 163, 121.

28. McLeod, L. D., Coon, C. D., Martin, S. A., Fehnel, S. E., \& Hays, R. D. (2011) Interpreting patient-reported outcome results: US FDA guidance and emerging methods. Expert Review of Pharmacoeconomics \& Outcomes Research, 11, 163-169.

\section{Publisher's Note}

Springer Nature remains neutral with regard to jurisdictional claims in published maps and institutional affiliations. 